\title{
Systematic Review of Surgical Approaches for Adrenal Tumors: Lateral Transperitoneal versus Posterior Retroperitoneal and Laparoscopic versus Robotic Adrenalectomy
}

\author{
Young Jun Chai, ${ }^{1,2}$ Hyungju Kwon, ${ }^{2,3}$ Hyeong Won Yu, ${ }^{2,3}$ Su-jin Kim, ${ }^{2,3}$ \\ June Young Choi, ${ }^{2,4}$ Kyu Eun Lee, ${ }^{2,3}$ and Yeo-Kyu Youn ${ }^{2,3}$ \\ ${ }^{1}$ Department of Surgery, Seoul National University Boramae Medical Center, 20 Boramae-ro 5-gil, Dongjak-gu, \\ Seoul 156-70, Republic of Korea \\ ${ }^{2}$ Cancer Research Institute, Seoul National University College of Medicine, 101 Daehak-ro, Jongno-gu, Seoul 110-744, Republic of Korea \\ ${ }^{3}$ Department of Surgery, Seoul National University Hospital and College of Medicine, 101 Daehak-ro, Jongno-gu, \\ Seoul 110-744, Republic of Korea \\ ${ }^{4}$ Department of Surgery, Seoul National University Bundang Hospital and College of Medicine, 300 Gumi-dong, Bundang-gu, \\ Seongnam 463-707, Republic of Korea \\ Correspondence should be addressed to Kyu Eun Lee; kyu.eun.lee.md@gmail.com
}

Received 4 November 2014; Accepted 5 December 2014; Published 17 December 2014

Academic Editor: Michael Horowitz

Copyright (C) 2014 Young Jun Chai et al. This is an open access article distributed under the Creative Commons Attribution License, which permits unrestricted use, distribution, and reproduction in any medium, provided the original work is properly cited.

\begin{abstract}
Background. Laparoscopic lateral transperitoneal adrenalectomy (LTA) has been the standard method for resecting benign adrenal gland tumors. Recently, however, laparoscopic posterior retroperitoneal adrenalectomy (PRA) has been more popular as an alternative method. This systematic review evaluates current evidence on adrenalectomy techniques, comparing laparoscopic LTA with PRA and laparoscopic adrenalectomy with robotic adrenalectomy. Methods. PubMed, Embase, and ISI Web of Knowledge databases were searched systematically for studies comparing surgical outcomes of laparoscopic LTA versus PRA and laparoscopic versus robotic adrenalectomy. The studies were evaluated according to the PRISMA statement. Results. Eight studies comparing laparoscopic PRA and LTA showed that laparoscopic PRA was superior or at least comparable to laparoscopic LTA in operation time, blood loss, pain score, hospital stay, and return to normal activity. Conversion rates and complication rates were similar. Six studies comparing robotic and laparoscopic adrenalectomy found that outcomes and complications were similar. Conclusion. Laparoscopic PRA was more effective than LTA, especially in reducing operation time and hospital stay, but there was no evidence showing that robotic adrenalectomy was superior to laparoscopic adrenalectomy. Cost reductions and further technical advances are needed for wider application of robotic adrenalectomy.
\end{abstract}

\section{Introduction}

Open transperitoneal adrenalectomy has been the gold standard of treatment for adrenal disease. According to the Nationwide Inpatient Sample [1], 83\% of adrenalectomies from 1998 through 2006 were performed using the open method, despite laparoscopic adrenalectomy shown to be successful in 1992 [2]. As conventional open adrenalectomy offers a wide surgical view and operative field, it is still preferred to laparoscopic adrenalectomy for large tumors and malignancies [3]. Laparoscopic adrenalectomy is not indicated for malignancy because it is associated with higher recurrence rates [4], although it could be performed safely in selected patients with isolated metastatic adrenal tumors [5].

More recently, however, laparoscopic procedures have been shown to be advantageous, with minimally invasive adrenalectomy replacing open adrenalectomy. Minimally invasive adrenalectomy results in less blood loss, earlier ambulation, shorter hospital stay, and faster return to normal activity [6]. These advantages were made possible by accumulated experience, advanced laparoscopic techniques, and better understanding of adrenal gland anatomy. At present, 
lateral transperitoneal adrenalectomy (LTA) has become the most widely utilized procedure for patients with benign adrenal disease. Other minimally invasive techniques include the anterior transperitoneal approach and the retroperitoneal (lateral or posterior) approach [7].

Since its introduction in 1995, posterior retroperitoneal adrenalectomy (PRA) has been utilized more frequently [8]. This technique consists of approaching the adrenal gland directly through the retroperitoneal space, while not breaching the peritoneum, resulting in a shorter operative time, less blood loss, less postoperative pain, and shorter hospital stay [9].

Although several studies have compared the outcomes of LTA and PRA, their overall results are inconclusive. Each study was retrospective in design and was conducted in a single center with different inclusion criteria. In addition, several meta-analyses have compared transperitoneal with retroperitoneal adrenalectomy [9-11]. These analyses, however, were not fully reliable, because the numbers of included studies and the study population were insufficient for metaanalyses. Furthermore, these analyses included older studies, which utilized different operation methods than those used currently or did not distinguish true PRA from lateral retroperitoneal adrenalectomy. This systematic review therefore compared surgical outcomes in patients undergoing LTA or PRA, as well as comparing outcomes in patients undergoing robotic or laparoscopic LTA or PRA.

\section{Methods}

2.1. Literature Search. The PubMed, Embase, and ISI Web of Knowledge databases were searched for studies, published in English after 2000, comparing laparoscopic LTA with PRA and robotic adrenalectomy with laparoscopic adrenalectomy. Using the criteria of the PRISMA statement, two authors (Young Jun Chai and Hyungju Kwon) independently searched the literature on 25 May 2014 for relevant studies [12]. Keywords for comparisons of laparoscopic LTA with included "lateral transperitoneal adrenalectomy," "laparoscopic transabdominal adrenalectomy," "laparoscopic transperitoneal adrenalectomy," "retroperitoneoscopic adrenalectomy", and "retroperitoneal adrenalectomy." Keywords for comparisons between robotic and laparoscopic LTA or PRA included "robotic adrenalectomy" and "robot-assisted adrenalectomy." References cited in relevant papers were also searched manually.

2.2. Inclusion and Exclusion Criteria. Clinical studies comparing LTA with PRA or laparoscopic with robotic adrenalectomy were reviewed by the two independent authors. Only single center studies that enrolled more than 20 patients were included. After reviewing operative methodology, studies that used the lateral retroperitoneal approach were excluded, allowing comparisons of LTA with true PRA. Studies that did not describe surgical procedures in detail, enabling categorization as LTA or PRA, were also excluded. In comparisons of laparoscopic and robotic adrenalectomy, studies that did not report the outcomes of LTA and PRA separately were excluded. If there were multiple reports from the same study patients, only the most recent or the largest study was included.

2.3. Data Extraction. Data collected by the two independent authors included the last name of the first author, study design, year of publication, patient characteristics, indications for LTA or PRA, operation time, blood loss, pain score, hospital stay, conversion rate, and characteristics of complications. Conversion to open surgery and conversion from robotic to laparoscopic surgery were counted together.

2.4. Surgical Procedures of Laparoscopic LTA. For LTA, the patient is placed in the lateral decubitus position with the affected side facing upward and the operative bed flexed just above the level of the iliac crest [13]. In general, three ports are made for left adrenalectomy, with one additional port required for right adrenalectomy to mobilize and lift the liver. Although the ports were commonly made at the umbilicus and the subcostal area in the anterior axillary and midclavicular lines, port sites could be modified at the discretion of the surgeon. All ports were inserted through the peritoneum, with the adrenal gland exposed by dissecting adjacent organs. The spleen, distal pancreas, and splenic flexure of the colon were detached from the retroperitoneum for left adrenalectomy, whereas the triangular ligament of the liver was dissected and rotated medially using a fan retractor for right adrenalectomy. After dividing the surrounding blood vessels, including the adrenal vein, the adrenal gland was placed in a plastic bag and retracted through a port site.

2.5. Surgical Procedures of Laparoscopic PRA. This procedure was performed as described [14]. Briefly, anesthesia was induced in a patient bed, and the patient was intubated and turned from the bed onto the operating table. The patient was placed in a prone position, lying on the abdomen with bent hip and knee joints. In general, three ports were required for this procedure. The first incision, about $1.5 \mathrm{~cm}$ in size, was made just below the tip of the twelfth rib, and the retroperitoneal space was bluntly dissected with a finger. Through this space, the second and third ports were made under direct vision $4-5 \mathrm{~cm}$ medially and laterally. After $\mathrm{CO}_{2}$ insufflation, perinephric fatty tissues were dissected from the posterior aspect of kidney, and the superior pole of the kidney was exposed. The inferior aspect of the adrenal gland was mobilized from the superior pole of the kidney. Adrenalectomy was completed by detaching the adrenal gland from adjacent structures and ligation of the adrenal vein. The resected adrenal gland is placed in a plastic bag and pulled out through the first incision site.

2.6. Procedures of Robotic LTA and Robotic PRA. Robotic adrenalectomy was firstly performed in 2000 [15]. The surgical procedures of robotic LTA and PRA are basically similar to those of laparoscopic LTA and PRA, such as patient position, port sites, use of $\mathrm{CO}_{2}$ gas, and specimen removal $[16,17]$. The patient is placed in a lateral decubitus position with lesion side upward (LTA) or in the prone jackknife position with bent hip and knee joints (PRA). After creation of pneumoperitoneum, to be used as a $12 \mathrm{~mm}$ camera port, 


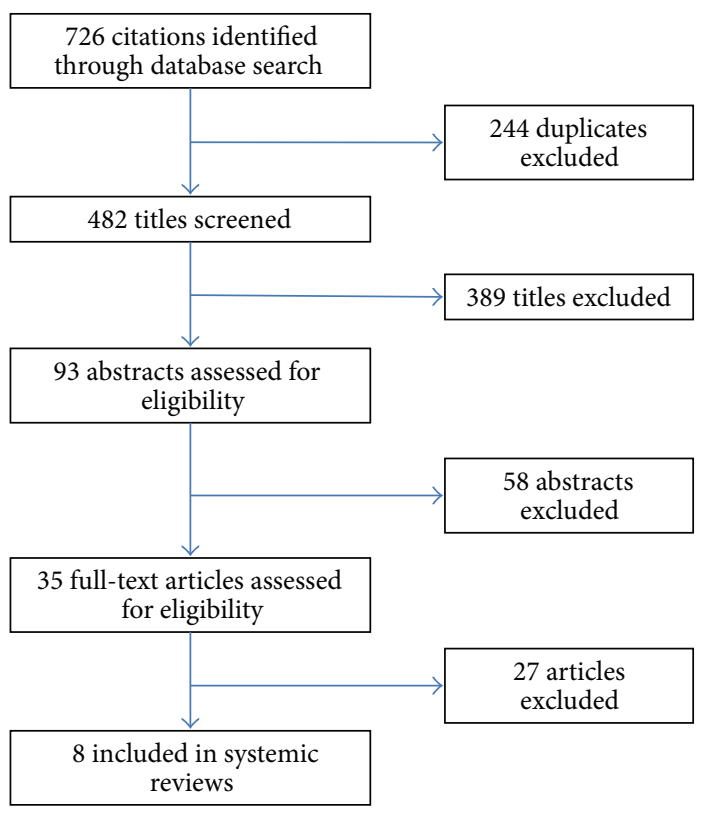

(a)

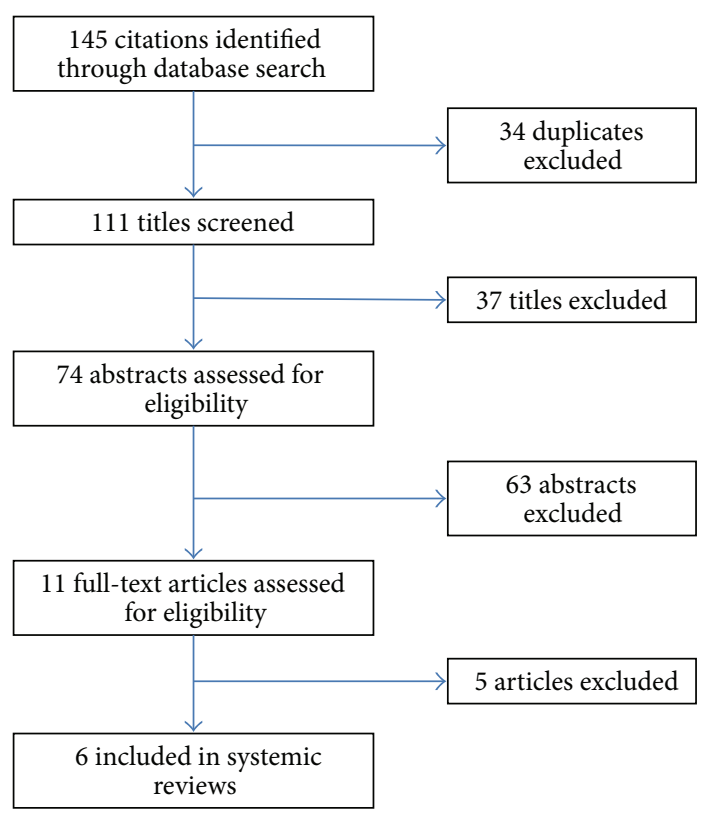

(b)

FIGURE 1: Flow charts showing selection of articles for systematic review. (a) Laparoscopic lateral transperitoneal adrenalectomy versus posterior retroperitoneoscopic adrenalectomy. (b) Laparoscopic adrenalectomy versus robotic adrenalectomy.

the other two or three $5 \mathrm{~mm}$ robotic ports are made. The robot is docked, with subsequent procedures basically identical to those of laparoscopic LTA and PRA.

2.7. Statistical Analysis. Results were analyzed with SPSS version 19 (SPSS, Inc., Chicago, IL, USA). Mean and median values with standard deviation were used for numeric data. Univariate analysis was performed using the Mann-Whitney $U$ test, chi-Square test, or Fisher's exact test, as warranted. $P<0.05$ was considered statistically significant.

\section{Results}

In comparing LTA and PRA, the literature search yielded 482 titles, after excluding duplicates (Figure 1). After screening for relevance, 93 abstracts were deemed eligible for review; of these, 58 were excluded as they were not original papers or did not meet inclusion criteria. Of the 35 full-text articles searched, eight were included in the analysis. In comparing laparoscopic adrenalectomy with robotic adrenalectomy, 111 titles were selected, after excluding duplicates. After screening the abstracts and full-texts, a total of six articles were included.

\subsection{Indications and Contraindications for Laparoscopic LTA} and PRA. Table 1 shows the size criteria and patient characteristics of the eight studies comparing laparoscopic LTA with true PRA [18-25]. In most of the studies, malignant tumors were considered a contraindication for laparoscopic surgery; however, LTA or PRA was successfully performed in patients with no evidence of invasion on preoperative imaging or when the lesion was an isolated metastasis [20,21]. Patients were deemed eligible for PRA if their tumors were less than
$8 \mathrm{~cm}$ in diameter, although size criteria were more liberal for LTA. There were no significant age differences in selecting LTA or PRA, but some studies reserved PRA for patients with lower body mass index (BMI) and smaller tumor size $[20,21,24]$.

3.2. Comparative Surgical Outcomes: Laparoscopic LTA versus Laparoscopic PRA. The surgical outcomes of patients undergoing LTA and PRA are shown in Table 2. PRA and LTA showed similar operation times in early studies [1821], although more recent studies reported that operation time was shorter for PRA than for LTA [22-24]. Four studies described intraoperative blood loss $[18,20,22,23]$, with one finding that blood loss was lower with PRA [22], and the other three reported no significant difference. Postoperative pain score was compared in one study [21], which found that pain scores on postoperative days one and three were significantly lower in the PRA than in the LTA group. Seven studies compared hospital stay after operation with the five most recent studies finding that mean hospital stay was shorter in patients who underwent PRA than LTA [21-25]. Time to return to work or days of convalescence were evaluated in two studies $[18,19]$, with one showing that PRA was associated with earlier return to normal activity [19], and the other reporting similar results in patients undergoing PRA and LTA [18].

3.3. Complications: Laparoscopic LTA versus Laparoscopic PRA. Complications of laparoscopic LTA and PRA are summarized in Table 3. Two studies reported conversion to open surgery or conversion from PRA to LTA $[18,20]$. In one, four patients were converted to open surgery from LTA and three from PRA. Of them, excessive operation time was the 


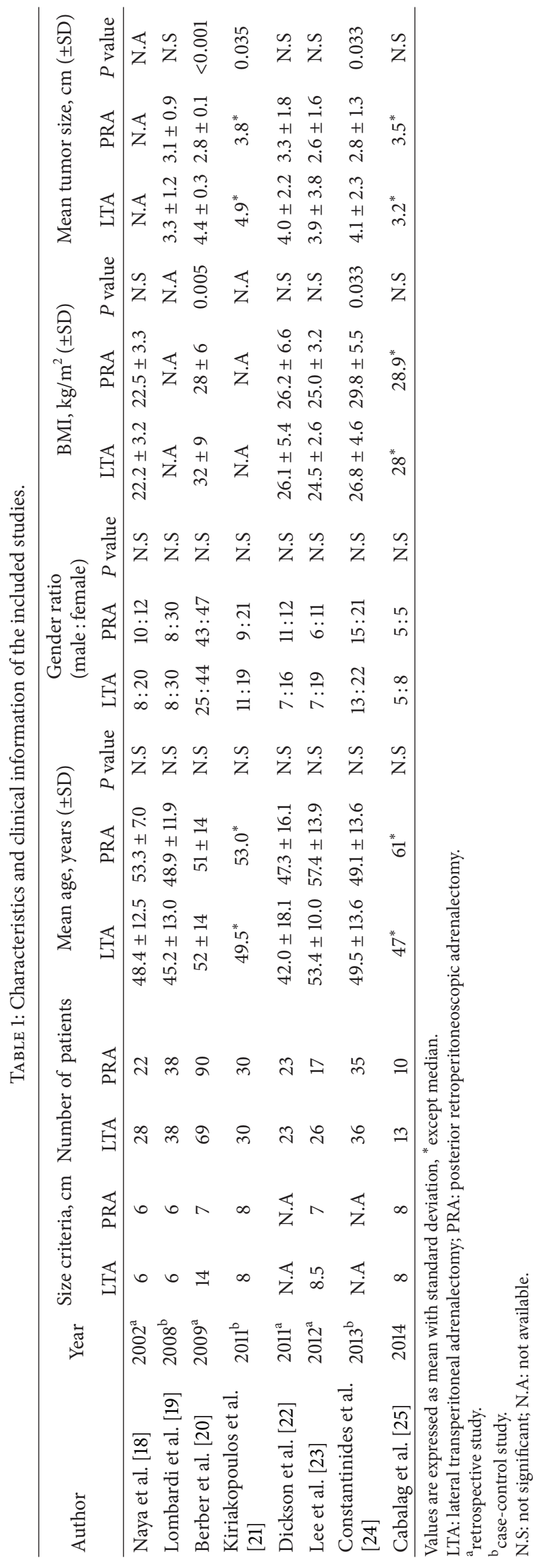




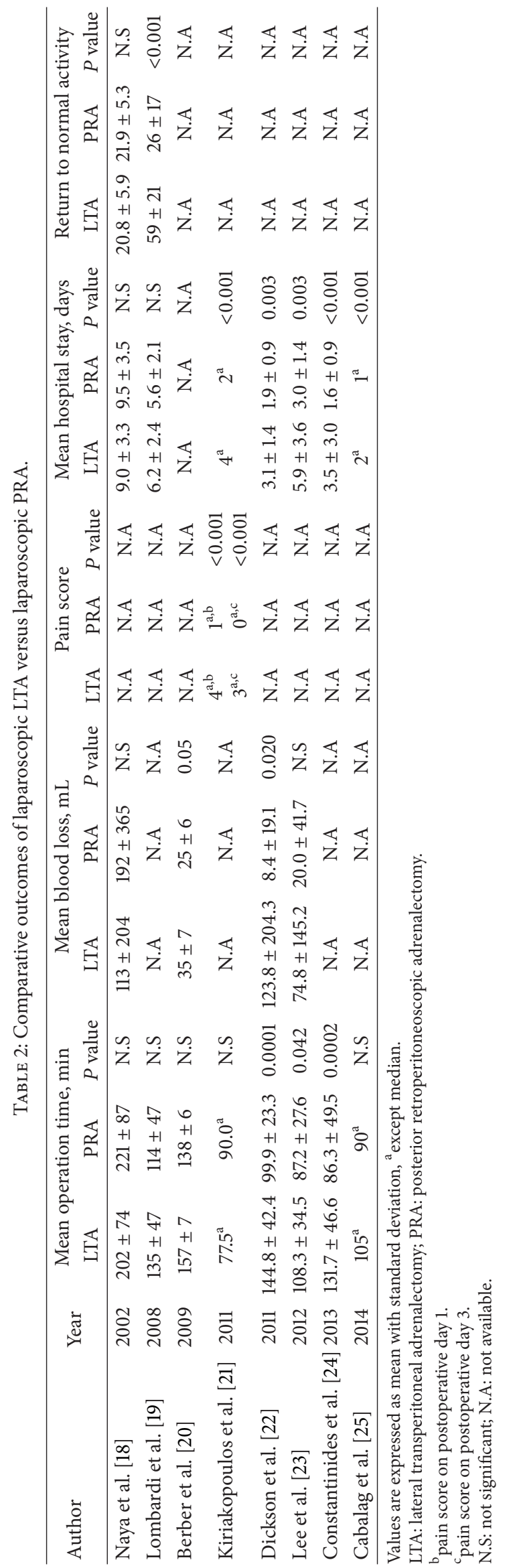


TABLE 3: Complications of laparoscopic LTA versus PRA.

\begin{tabular}{|c|c|c|c|c|c|c|c|c|}
\hline \multirow[t]{2}{*}{ Author } & \multicolumn{2}{|c|}{ Conversion, $n(\%)$} & \multicolumn{2}{|c|}{$\begin{array}{l}\text { Postoperative bleeding } \\
\text { requiring surgery, } n(\%)\end{array}$} & \multicolumn{2}{|c|}{ Mortality, $n(\%)$} & \multicolumn{2}{|c|}{ Others, $n(\%)$} \\
\hline & LTA & PRA & LTA & LTA & LTA & PRA & LTA & PRA \\
\hline Naya et al. [18] & $4^{\mathrm{a}}$ & $3^{\mathrm{b}}$ & 0 & 0 & 0 & 0 & 2 & 2 \\
\hline Lombardi et al. [19] & 0 & 0 & 1 & 0 & 0 & 0 & 0 & 2 \\
\hline Berber et al. [20] & $2^{c}$ & $2^{\mathrm{d}}$ & 0 & 0 & $2^{\mathrm{e}}$ & 0 & 0 & 2 \\
\hline Kiriakopoulos et al. [21] & 0 & 0 & 0 & 0 & 0 & 0 & 0 & 4 \\
\hline Dickson et al. [22] & 0 & 0 & 2 & 0 & 0 & 0 & 0 & 2 \\
\hline Lee et al. [23] & 0 & 0 & 0 & 0 & 0 & 0 & 0 & 0 \\
\hline Constantinides et al. [24] & 0 & 0 & 0 & 0 & 0 & 0 & 3 & 1 \\
\hline Cabalag et al. [25] & 0 & 0 & 0 & 0 & 0 & 0 & 4 & 1 \\
\hline Total & $6 / 263(2.3)$ & $5 / 265(1.9)$ & $3 / 263(1.1)$ & $0 / 265(0)$ & $2 / 263(0.7)$ & $0 / 265(0)$ & $9 / 263(3.4)$ & $14 / 265(5.3)$ \\
\hline
\end{tabular}

LTA: lateral transperitoneal adrenalectomy; PRA: posterior retroperitoneoscopic adrenalectomy.

a open conversion due to diaphragm injury or excessive operation time, ${ }^{b}$ open conversion due to intercostal artery bleeding or excessive operation time, ${ }^{c}$ open conversion due to bleeding, ${ }^{\mathrm{d}}$ conversion from PRA to LTA due to inadequate establishment of retroperitoneal space, and ${ }^{\mathrm{e}}$ cardiac and pulmonary complication.

cause in 3 LTA and in 2 PRA cases [18]. One patient who underwent LTA required conversion due to a diaphragm injury and one who underwent PRA required conversion due to intercostal artery bleeding caused by trocar insertion. In the other study, two patients were converted to open surgery from LTA due to bleeding from the adrenal vein, and two were converted from PRA to LTA due to inadequate establishment of retroperitoneal space during the initial learning curve for PRA [20]. Total conversion rates were 2.3\% (6/263) for LTA and $1.9 \%(5 / 265)$ for PRA.

Two patients who underwent LTA died from cardiac and pulmonary complication [20], making the mortality rates $0.7 \%$ and $0 \%$ in patients undergoing LTA and PRA, respectively. Postoperative bleeding (1.1\%; 3/263), diaphragm injury $(0.4 \% ; 1 / 263)$, pulmonary embolism $(0.8 \% ; 2 / 263)$, port site incisional hernia $(0.4 \% ; 1 / 263)$, postoperative ileus $(0.4 \% ; 1 / 263)$, pneumonia $(0.4 \% ; 1 / 263)$, and major cardiac arrhythmia $(0.4 \% ; 1 / 263)$ occurred only in the LTA group. Complications in patients undergoing PRA included thrombophlebitis $(0.4 \% ; 1 / 265)$, temporary hypoesthesia $(0.4 \%$; $1 / 265)$, neuralgia $(0.8 \% ; 2 / 265)$, paresthesia $(0.4 \% ; 1 / 265)$, lateral abdominal swelling $(1.5 \% ; 4 / 265)$, pneumothorax $(0.4 \% ; 1 / 265)$, pleural effusion $(0.4 \% ; 1 / 265)$, and stroke $(0.4 \% ; 1 / 265)$. Wound infection occurred in one patient each undergoing LTA and PRA (0.4\% each). Total complication rates were $5.3 \%(14 / 263)$ for LTA and 5.3\% (14/265) for PRA.

3.4. Comparative Surgical Outcomes: Laparoscopic versus Robotic Adrenalectomy. Since the introduction of the da Vinci surgical robot system (Intuitive Surgical, Inc., Sunnyvale, CA, USA) for cardiac surgery in 1998, its use has expanded to many other types of surgery, including endocrine surgery. The robotic system was found to overcome some of the limitations of laparoscopic surgery, including its inflexibility, two-dimensional operative view, and fatigue, by providing a tremor-free endowrist, three-dimensional imaging, and excellent ergonomics $[26,27]$. Despite its high cost, many surgeons have applied robotic surgery to LTA and PRA to maximize surgical efficiency.
Six studies met the inclusion criteria; their results were shown in Table 4 [28-33]. During the early period of robot use, as shown in the first two studies, operation times were significantly longer for robotic LTA than for laparoscopic LTA $[28,29]$. More recently, however, as shown in the four later studies, there were no differences in operation time between laparoscopic and robotic LTA [30-33]. Two studies reported that blood loss was lower for robotic LTA $[29,33]$, and one study reported less pain on postoperative day one for robotic LTA [30]. Hospital stay was similar in both groups, although one study reported shorter hospital stay in the robotic group [31].

3.5. Complications: Laparoscopic versus Robotic Adrenalectomy. Table 5 shows complications of laparoscopic and robotic adrenalectomy. The most common cause of conversion from robotic to laparoscopic adrenalectomy or open laparotomy was bleeding $(2.2 \% ; 4 / 186)$. Other causes included inadequate visualization $(1.6 \% ; 3 / 186)$, prolonged operation time $(0.5 \% ; 1 / 186)$, and tumor adhesion $(0.5 \%$; 1/186). Patients with complications, such as bleeding or tumor invasion, were converted to open laparotomy, whereas those with minor problems, such as inadequate visualization, were converted to laparoscopic procedures. Overall conversion rates were similar for robotic and laparoscopic adrenalectomy.

There were no unique complications related to robotic adrenalectomy. Types of complications in patients undergoing robotic procedures included pneumonia $(1.6 \% ; 3 / 186)$, wound problems $(1.6 \% ; 3 / 186)$, urinary tract infection $(0.5 \%$; $1 / 186)$, postoperative ileus $(0.5 \% ; 1 / 186)$, chylous ascites $(0.5 \%$; $1 / 186)$, hyponatremia $(0.5 \% ; 1 / 186)$, vomiting $(0.5 \% ; 1 / 186)$, atrial fibrillation $(0.5 \% ; 1 / 186)$, and postoperative bleeding requiring blood transfusion $(0.5 \% ; 1 / 186)$.

\section{Discussion}

The present systematic review suggests that PRA may be superior to LTA in terms of shorter operation time and 


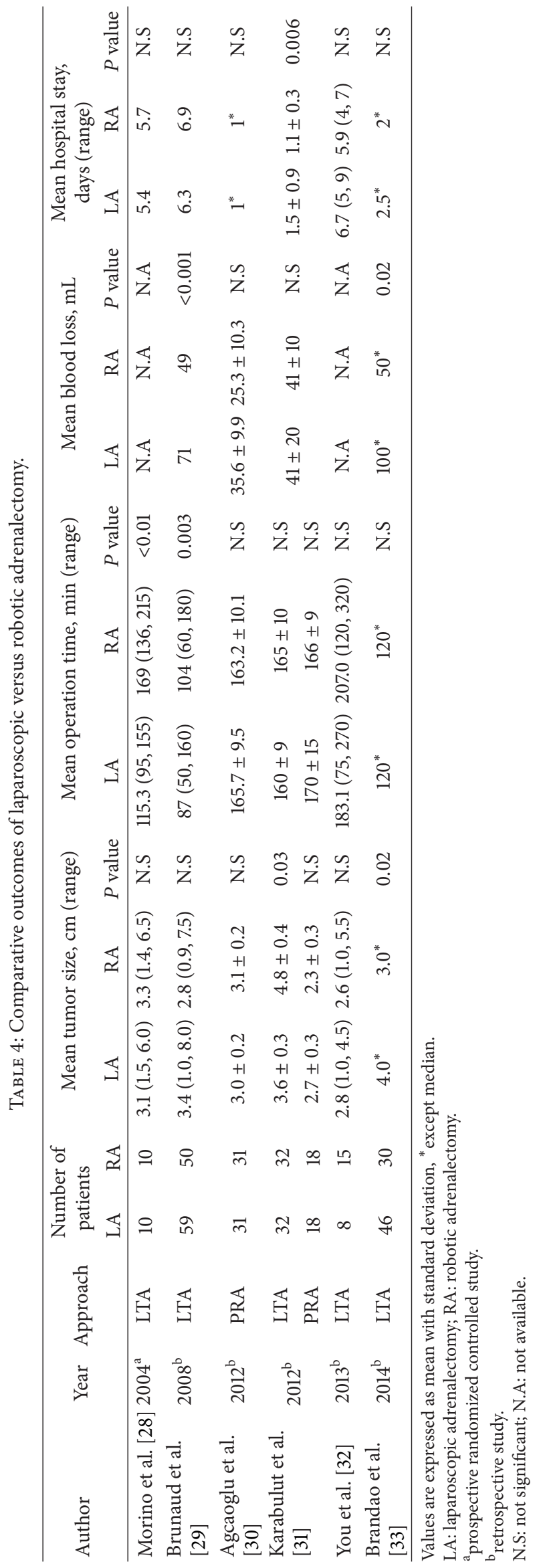




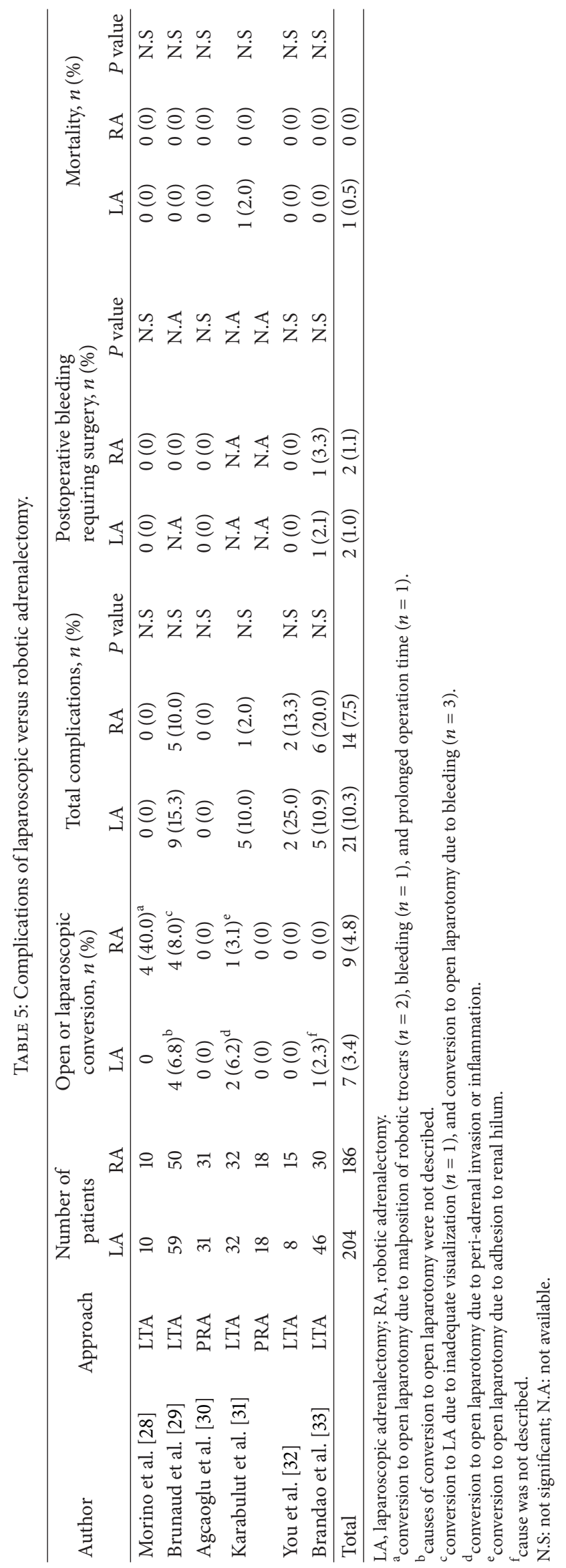


hospital stay, as well as reduced blood loss and pain score. The superiority of PRA may be due to the location of the adrenal glands in the posterior aspect of the retroperitoneum. PRA directly approaches this space, enabling adrenalectomy without collateral damage to adjacent intra-abdominal organs, which must be dissected and mobilized during LTA or open adrenalectomy [34]. In consequence, recovery of bowel movement was faster and postoperative ileus rarely observed in the patients who underwent PRA. Furthermore, PRA is feasible in patients with a previous history of abdominal surgery, as well as being suitable for bilateral adrenalectomy. Reports of good intra- and postoperative outcomes of PRA have made this method more popular. However, PRA is not easy for beginners to learn because surgeons are not familiar with this anatomic view of the retroperitoneal space. Thus training in the use of PRA requires a substantial amount of time. PRA is also difficult to perform in patients with tumors larger than $7-8 \mathrm{~cm}$ and in patients with a high BMI [14].

In three of the eight studies enrolled in this review, operation time was significantly shorter for laparoscopic PRA than LTA; the other five studies showed no between group differences in operation times. The shorter operation time in PRA was most likely due to the smaller extent of dissection required. Moreover, PRA was performed by surgeons skilled in the laparoscopic technique for LTA, allowing them to overcome the learning curve for PRA more easily. A comparison of the first 50 operations performed by the developers of PRA and 50 operations performed by a surgical team that learned PRA from the developers found that the learning curve was shorter in the latter group, suggesting that comprehensive training resulted in a shorter operation time and a lower conversion rate [35]. Hence, PRA is safe when performed by surgeons with LTA experience or those who undergo proper PRA training.

In all five studies reported since 2011, hospital stay was shorter in the PRA than in the LTA group [21-25], in agreement with the results of a meta-analysis comparing LTA and PRA [9]. Shorter hospital stay may be associated with reduced pain, as the median visual analog pain scores in patients undergoing PRA and LTA were reported as 0 and 3, respectively [21]. Another parameter indicating the amount of postoperative pain is analgesic use during hospitalization, which was evaluated in three studies [19, 23, 25]. Analgesic use was lower in the PRA group in two of these studies [23, 25] and equal in the third [19].

Conversion rates of LTA and PRA to open laparotomy were $2.3 \%(6 / 263)$ and $1.9 \%(5 / 265)$, respectively, but the reasons for conversion differed. Conversion in LTA was associated with diaphragm injury or vascular injury, whereas conversion in PRA was associated with inadequate preparation of the retroperitoneal working space due to surgeon inexperience. A unique complication associated only with PRA was neuromuscular pain related to subcostal nerve injury [36]. The subcostal nerve passes below the twelfth rib and the injury to this nerve commonly occurs during open posterior adrenalectomy, leading to chronic incision-related back pain [37]. Likewise, trocar insertion in this area during PRA can cause nerve damage. The incidence of nerve damage in the largest PRA series was reported to be $9 \%$, but was temporary in most patients [14]. Although concerns have been raised regarding the high $\mathrm{CO}_{2}$ pressure required for PRA [38], there were no complications associated with high pressure, except for air embolism in one patient causing a stroke [24]. In particular, even in the study comparing LTA with PRA in patients with pheochromocytoma, there were no significant hemodynamic differences, such as intraoperative hypertension, hypotension, and vasoactive medication use [22].

Early comparisons of robotic and laparoscopic LTA found that operation time was longer for the former [28, 29]. However, operation time is largely dependent on the surgeon's experience. A detailed analysis of operation time in 50 patients undergoing robotic and 59 undergoing laparoscopic LTA found that the learning curve for robotic LTA was 20 cases, with no difference after the learning curve between operation times for robotic and laparoscopic LTA [29]. Moreover, previous experience with laparoscopic LTA or assistance from a staff surgeon contributed to reductions in operation time. Another report evaluating 100 robotic adrenalectomies found that surgeon's experience and first assistant level were independent predictors of longer operation time [16]. Notably, operation time of robotic LTA was not associated with high BMI $\left(>30 \mathrm{~kg} / \mathrm{m}^{2}\right)$ or large tumor size $(>55 \mathrm{~mm})$, whereas operation times of laparoscopic LTA were increased under those conditions. In comparative studies published since 2012, there were no significant differences in operation times between robotic and laparoscopic adrenalectomy [3033]. A comparison of robotic and laparoscopic PRA showed that pain score on postoperative day 1 was significantly lower in the robotic group (2.5 versus $4.2, P=0.008$ ) [30]. The lower pain score in the robotic PRA group was attributed to reduced manipulation of the incision, with fewer instrument changes and less pressure exerted by the surgical team on the patient's back. However, the mean pain scores became similar on postoperative day 14.

Robotic surgery with a similar operation time and ergonomic improvements relative to laparoscopic surgery is attractive for surgeons, since robotic procedures offer more delicate dissection using endowrist and a magnified view. Robotic adrenalectomy may have advantages in maintaining a vascularized remnant during cortical sparing adrenalectomy [39]. However, it is unclear whether the substantial additional cost of the robotic procedure $(\$ 3,466$ versus $\$ 2,737$ ) is justified, since outcomes are equivalent [28]. No randomized controlled trial has compared robotic adrenalectomy with laparoscopic adrenalectomy, and no study to date has shown that robotic adrenalectomy is superior to laparoscopic adrenalectomy in postoperative outcomes. Thus, the costs of the initial purchase of the robot and instruments, as well as its maintenance, are obstacles to the expansion of robotic adrenalectomy.

Several promising technical applications can be easily adapted for robotic adrenalectomy. For example, after indocyanine green dye injection, robotic partial adrenalectomy can be performed safely using the da Vinci SI system with FireFly scopes, a light source, and a software upgrade [40]. Indocyanine green dye can easily delineate the tumor 
margins, allowing uninvolved adrenal tissue to be saved. Other promising applications include telemonitoring and telerobotic surgery. Telemonitoring may be effective method in performing a new surgical technique, allowing remote monitoring by a trained surgeon [41]. Robotic surgery is more suitable for telemonitoring because robotic systems offer three-dimensional images, and the monitoring surgeons can better view patient anatomy. Robotic adrenalectomy can also be better optimized for new technical advances such as telesurgery or image-guided surgery and promising surgical modalities.

\section{Conclusions}

PRA may be superior to LTA, as shown by shorter operation times and hospital stay, and can be performed safely after surgeons overcome the learning curve. Randomized controlled trials comparing these two techniques are necessary to objectively evaluate them, excluding selection bias and bias related to differences in surgeons' experiences with these techniques. Current data suggest that robotic adrenalectomy is safe and feasible but have not shown definite advantages over laparoscopic adrenalectomy to date. Cost reductions or further improvements in surgical outcomes are necessary to expand the use of robotic adrenalectomy.

\section{Conflict of Interests}

The authors declare that there is no conflict of interests regarding the publication of this paper.

\section{References}

[1] M. M. Murphy, E. R. Witkowski, S. C. Ng et al., "Trends in adrenalectomy: a recent national review," Surgical Endoscopy and Other Interventional Techniques, vol. 24, no. 10, pp. 25182526, 2010.

[2] M. Gagner, A. Lacroix, and E. Bolte, "Laparoscopic adrenalectomy in Cushing's syndrome and pheochromocytoma," The New England Journal of Medicine, vol. 327, no. 14, p. 1033, 1992.

[3] J. G. Bittner IV, V. M. Gershuni, B. D. Matthews, J. F. Moley, and L. M. Brunt, "Risk factors affecting operative approach, conversion, and morbidity for adrenalectomy: a single-institution series of 402 patients," Surgical Endoscopy, vol. 27, no. 7, pp. 2342-2350, 2013.

[4] A. B. Cooper, M. A. Habra, E. G. Grubbs et al., "Does laparoscopic adrenalectomy jeopardize oncologic outcomes for patients with adrenocortical carcinoma?" Surgical Endoscopy, vol. 27, no. 11, pp. 4026-4032, 2013.

[5] J. Y. Chen, A. Ardestani, and A. Tavakkoli, "Laparoscopic adrenal metastasectomy: appropriate, safe, and feasible," Surgical Endoscopy, vol. 28, no. 3, pp. 816-820, 2014.

[6] G. Guazzoni, F. Montorsi, A. Bocciardi et al., "Transperitoneal laparoscopic versus open adrenalectomy for benign hyperfunctioning adrenal tumors: a comparative study," The Journal of Urology, vol. 153, no. 5, pp. 1597-1600, 1995.

[7] A. A. Gumbs and M. Gagner, "Laparoscopic adrenalectomy," Best Practice \& Research Clinical Endocrinology \& Metabolism, vol. 20, no. 3, pp. 483-499, 2006.
[8] S. Mercan, R. Seven, S. Ozarmagan, and S. Tezelman, "Endoscopic retroperitoneal adrenalectomy," Surgery, vol. 118, no. 6, pp. 1071-1076, 1995.

[9] V. A. Constantinides, I. Christakis, P. Touska, and F. F. Palazzo, "Systematic review and meta-analysis of retroperitoneoscopic versus laparoscopic adrenalectomy," British Journal of Surgery, vol. 99, no. 12, pp. 1639-1648, 2012.

[10] G. Nigri, A. S. Rosman, N. Petrucciani et al., "Meta-analysis of trials comparing laparoscopic transperitoneal and retroperitoneal adrenalectomy," Surgery, vol. 153, no. 1, pp. 111-119, 2013.

[11] W. Chen, F. Li, D. Chen et al., "Retroperitoneal versus transperitoneal laparoscopic adrenalectomy in adrenal tumor: a metaanalysis," Surgical Laparoscopy, Endoscopy \& Percutaneous Techniques, vol. 23, no. 2, pp. 121-127, 2013.

[12] D. Moher, A. Liberati, J. Tetzlaff, and D. G. Altman, "Preferred reporting items for systematic reviews and meta-analyses: the PRISMA statement," PLoS Medicine, vol. 6, no. 7, Article ID e1000097, 2009.

[13] Q.-Y. Duh, A. E. Siperstein, O. H. Clark et al., "Laparoscopic adrenalectomy. Comparison of the lateral and posterior approaches," Archives of Surgery, vol. 131, no. 8, pp. 870-876, 1996.

[14] M. K. Walz, P. F. Alesina, F. A. Wenger et al., "Posterior retroperitoneoscopic adrenalectomy-results of 560 procedures in 520 patients," Surgery, vol. 140, no. 6, pp. 943-950, 2006.

[15] W. Bentas, M. Wolfram, R. Bräutigam, and J. Binder, "Laparoscopic transperitoneal adrenalectomy using a remote-controlled robotic surgical system," Journal of Endourology, vol. 16, no. 6, pp. 373-376, 2002.

[16] L. Brunaud, A. Ayav, R. Zarnegar et al., "Prospective evaluation of 100 robotic-assisted unilateral adrenalectomies," Surgery, vol. 144, no. 6, pp. 995-1001, 2008.

[17] E. Berber, J. Mitchell, M. Milas, and A. Siperstein, "Robotic posterior retroperitoneal adrenalectomy: operative technique," Archives of Surgery, vol. 145, no. 8, pp. 781-784, 2010.

[18] Y. Naya, M. Nagata, T. Ichikawa et al., "Laparoscopic adrenalectomy: comparison of transperitoneal and retroperitoneal approaches," BJU International, vol. 90, no. 3, pp. 199-204, 2002.

[19] C. P. Lombardi, M. Raffaelli, C. de Crea et al., "Endoscopic adrenalectomy: is there an optimal operative approach? Results of a single-center case-control study," Surgery, vol. 144, no. 6, pp. 1008-1015, 2008.

[20] E. Berber, G. Tellioglu, A. Harvey, J. Mitchell, M. Milas, and A. Siperstein, "Comparison of laparoscopic transabdominal lateral versus posterior retroperitoneal adrenalectomy," Surgery, vol. 146, no. 4, pp. 621-626, 2009.

[21] A. Kiriakopoulos, K. P. Economopoulos, E. Poulios, and D. Linos, "Impact of posterior retroperitoneoscopic adrenalectomy in a tertiary care center: a paradigm shift," Surgical Endoscopy, vol. 25, no. 11, pp. 3584-3589, 2011.

[22] P. V. Dickson, G. C. Alex, E. G. Grubbs et al., "Posterior retroperitoneoscopic adrenalectomy is a safe and effective alternative to transabdominal laparoscopic adrenalectomy for pheochromocytoma," Surgery, vol. 150, no. 3, pp. 452-458, 2011.

[23] C. R. Lee, M. K. Walz, S. Park et al., "A comparative study of the transperitoneal and posterior retroperitoneal approaches for laparoscopic adrenalectomy for adrenal tumors," Annals of Surgical Oncology, vol. 19, no. 8, pp. 2629-2634, 2012.

[24] V. A. Constantinides, I. Christakis, P. Touska, K. Meeran, and F. Palazzo, "Retroperitoneoscopic or laparoscopic adrenalectomy? A single-centre UK experience," Surgical Endoscopy, vol. 27, no. 11, pp. 4147-4152, 2013. 
[25] M. S. Cabalag, G. B. Mann, A. Gorelik, and J. A. Miller, "Comparison of outcomes after laparoscopic versus posterior retroperitoneoscopic adrenalectomy: a pilot study," Surgical Laparoscopy, Endoscopy \& Percutaneous Techniques, vol. 24, no. 1, pp. 62-66, 2014.

[26] C. N. Gutt, T. Oniu, A. Mehrabi, A. Kashfi, P. Schemmer, and M. W. Büchler, "Robot-assisted abdominal surgery," British Journal of Surgery, vol. 91, no. 11, pp. 1390-1397, 2004.

[27] M. A. Savitt, G. Gao, A. P. Furnary, J. Swanson, H. L. Gately, and J. R. Handy, "Application of robotic-assisted techniques to the surgical evaluation and treatment of the anterior mediastinum," The Annals of Thoracic Surgery, vol. 79, no. 2, pp. 450-455, 2005.

[28] M. Morino, G. Benincà, G. Giraudo, G. M. del Genio, F. Rebecchi, and C. Garrone, "Robot-assisted vs laparoscopic adrenalectomy: a prospective randomized controlled trial," Surgical Endoscopy, vol. 18, no. 12, pp. 1742-1746, 2004.

[29] L. Brunaud, L. Bresler, A. Ayav et al., "Robotic-assisted adrenalectomy: what advantages compared to lateral transperitoneal laparoscopic adrenalectomy?" The American Journal of Surgery, vol. 195, no. 4, pp. 433-438, 2008.

[30] O. Agcaoglu, S. Aliyev, K. Karabulut, A. Siperstein, and E. Berber, "Robotic vs laparoscopic posterior retroperitoneal adrenalectomy," Archives of Surgery, vol. 147, no. 3, pp. 272-275, 2012.

[31] K. Karabulut, O. Agcaoglu, S. Aliyev, A. Siperstein, and E. Berber, "Comparison of intraoperative time use and perioperative outcomes for robotic versus laparoscopic adrenalectomy," Surgery, vol. 151, no. 4, pp. 537-542, 2012.

[32] J. Y. You, H. Y. Lee, G. S. Son, J. B. Lee, J. W. Bae, and H. Y. Kim, "Comparison of robotic adrenalectomy with traditional laparoscopic adrenalectomy with a lateral transperitoneal approach: a single-surgeon experience," The International Journal of Medical Robotics and Computer Assisted Surgery, vol. 9, no. 3, pp. 345350, 2013.

[33] L. F. Brandao, R. Autorino, H. Zargar et al., "Robot-assisted laparoscopic adrenalectomy: step-by-step technique and comparative outcomes," European Urology, vol. 66, no. 5, pp. 898905, 2014.

[34] M. K. Walz, K. Peitgen, R. Hoermann, R. M. Giebler, K. Mann, and F. W. Eigler, "Posterior retroperitoneoscopy as a new minimally invasive approach for adrenalectomy: results of 30 adrenalectomies in 27 patients," World Journal of Surgery, vol. 20, no. 7, pp. 769-774, 1996.

[35] M. Barczyński, A. Konturek, F. Gołkowski et al., "Posterior retroperitoneoscopic adrenalectomy: a comparison between the initial experience in the invention phase and introductory phase of the new surgical technique," World Journal of Surgery, vol. 31, no. 1, pp. 65-71, 2007.

[36] A. E. Siperstein, E. Berber, K. L. Engle, Q.-Y. Duh, and O. H. Clark, "Laparoscopic posterior adrenalectomy: technical considerations," Archives of Surgery, vol. 135, no. 8, pp. 967-971, 2000.

[37] J. F. Buell, H. R. Alexander, J. A. Norton, K. C. Yu, and D. L. Fraker, "Bilateral adrenalectomy for Cushing's syndrome: anterior versus posterior surgical approach," Annals of Surgery, vol. 225, no. 1, pp. 63-68, 1997.

[38] R. M. Giebler, M. K. Walz, K. Peitgen, and R. U. Scherer, "Hemodynamic changes after retroperitoneal CO2 insufflation for posterior retroperitoneoscopic adrenalectomy," Anesthesia \& Analgesia, vol. 82, no. 4, pp. 827-831, 1996.

[39] P. V. Dickson, G. C. Alex, E. G. Grubbs, C. Jimenez, J. E. Lee, and N. D. Perrier, "Robotic-assisted retroperitoneoscopic adrenalectomy: making a good procedure even better," American Surgeon, vol. 79, no. 1, pp. 84-89, 2013.

[40] T. B. Manny, A. S. Pompeo, and A. K. Hemal, "Robotic partial adrenalectomy using indocyanine green dye with near-infrared imaging: the initial clinical experience," Urology, vol. 82, no. 3, pp. 738-742, 2013.

[41] S. Treter, N. Perrier, J. A. Sosa, and S. Roman, “Telementoring: a multi-institutional experience with the introduction of a novel surgical approach for adrenalectomy," Annals of Surgical Oncology, vol. 20, no. 8, pp. 2754-2758, 2013. 


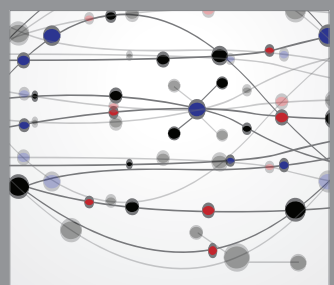

The Scientific World Journal
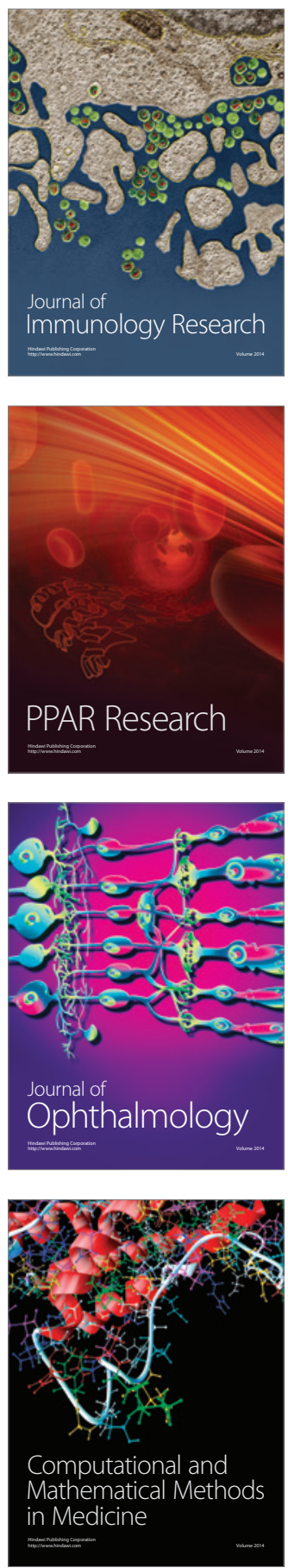

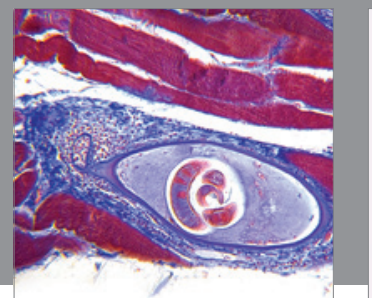

Gastroenterology

Research and Practice
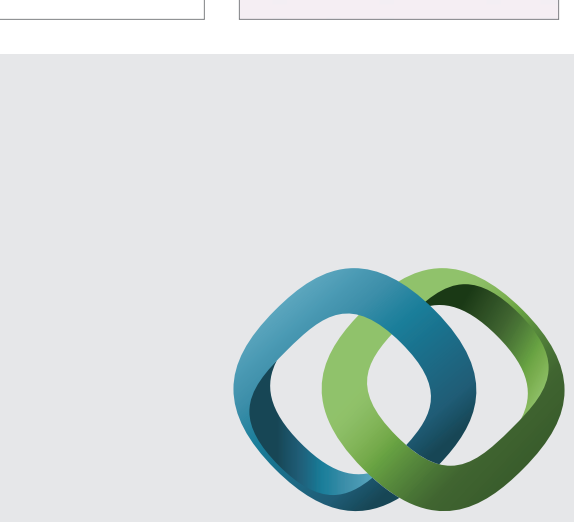

\section{Hindawi}

Submit your manuscripts at

http://www.hindawi.com
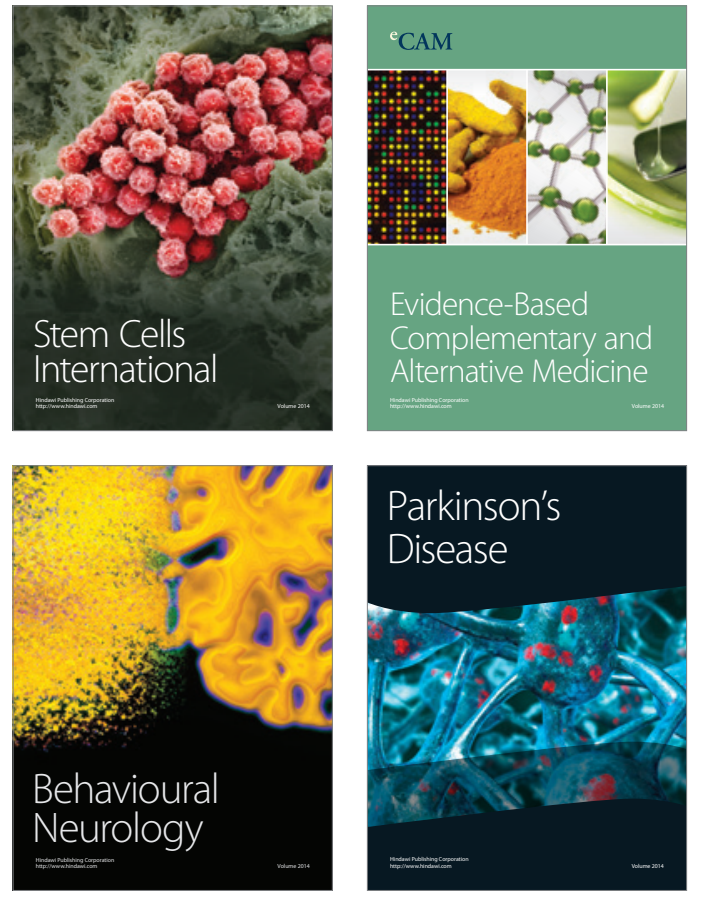
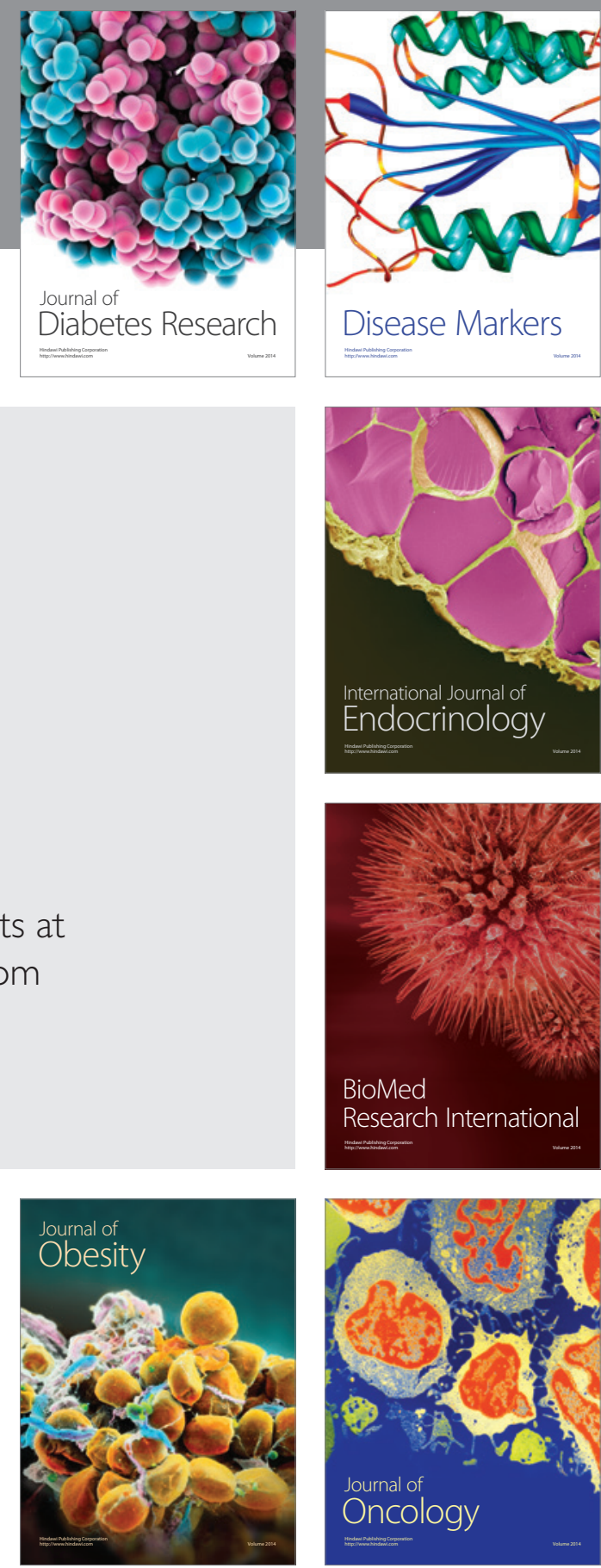

Disease Markers
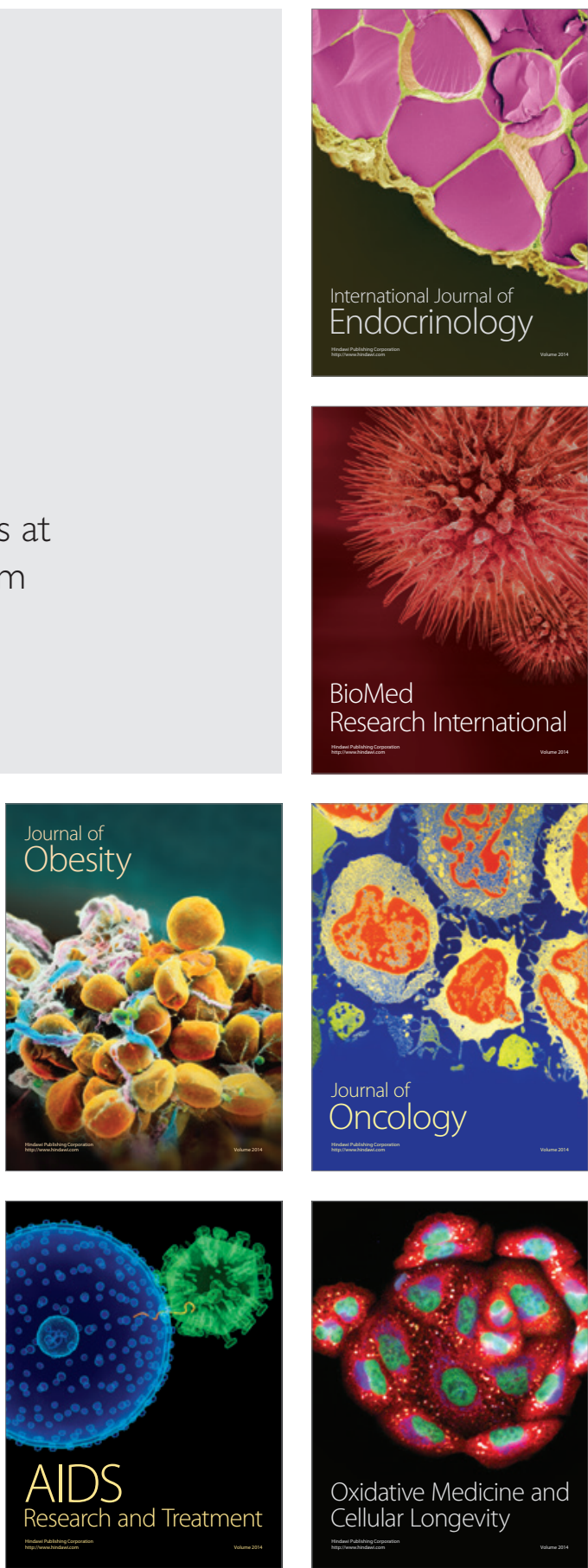